\title{
Action-specific effects underwater
}

\author{
Jessica K Witt, Donald M Schuck, J Eric T Taylor \\ Department of Psychological Sciences, Purdue University, West Lafayette, IN 47907, USA; \\ e-mail: jkwitt@purdue.edu \\ Received 18 January 2011, in revised form 18 May 2011; published online 6 July 2011
}

\begin{abstract}
Action-specific effects on perception are apparent in terrestrial environments. For example, targets that require more effort to walk, jump, or throw to look farther away than when the targets require less effort. Here, we examined whether action-specific effects would generalize to an underwater environment. Instead, perception might be geometrically precise, rather than action-specific, in an environment that is novel from an evolutionary perspective. We manipulated ease to swim by giving participants swimming flippers or taking them away. Those who estimated distance while wearing the flippers judged underwater targets to be closer than did participants who had taken them off. In addition, participants with better swimming ability judged the targets to be closer than did those with worse swimming ability. These results suggest perceived distance underwater is a function of the perceiver's ability to swim to the targets.
\end{abstract}

\section{Introduction}

Action-specific effects suggest that perception expresses the relationship between the surrounding environment and the perceiver's ability to act (eg Witt 2011a), just as Gibson (1979) theorized. For example, objects look closer and smaller when they are easier to reach and grasp (see below). The action-specific account of perception has been tested only in terrestrial environments, which are the environments in which our perceptual and motor systems likely evolved. Here, we examined if action-specific effects will also occur in environments that are novel from an evolutionary perspective. In such novel environments, perception may attempt to recover the precise geometric properties of the environment rather than to scale aspects of the environment to the perceiver's ability to act.

Action-specific effects may not be apparent in all aspects of the perceived environment. As an extreme example, the perceived size of the Moon is not likely influenced by action abilities. In the case of perceiving objects such as the Moon, perception must rely on arbitrary metrics not grounded in the body and its abilities (Proffitt and Linkenauger, in press). The question addressed here is whether perception in aquatic environments involves action-specific processes. To examine this question, we investigated if changes in the ability to swim influence underwater distance perception.

Above ground, there are several indications that perceivers see the surrounding environment in terms of their abilities. The ground plane looks steeper and distances look farther to perceivers who would have to exert more effort to traverse the space (Bhalla and Proffitt 1999; Lessard et al 2009; Proffitt et al 2003; Stefanucci et al 2005; Witt et al 2004, 2010). Objects placed on a tabletop look closer and smaller when they are easier to reach and grasp (Linkenauger et al 2009, in press; Witt 2011b; Witt et al 2005). When perceivers intend to grasp, objects of the correct orientation and size are detected more quickly and accurately than when intending to point or perform a different kind of grasp (Bekkering and Neggers 2002; Gutteling et al 2011; Symes et al 2008). After more successful sports performance, targets look bigger and slower 
(Cañal-Bruland and van der Kamp 2009; Witt and Dorsch 2009; Witt and Proffitt 2005; Witt and Sugovic 2010) and obstacles look smaller (Witt and Sugovic 2010). In summary, the surrounding environment is perceived in terms of the perceiver's ability to perform the intended action.

Here, we examined if action-specific effects generalize to aquatic environments. Distance perception underwater is typically overestimated relative to the same distances above ground (Luria and Kinney 1970; Luria et al 1967; Ross 1967). Researchers attribute this overestimation to optical factors such as reduced brightness contrast or seemingly increased aerial perspective. However, above ground, reduced cue settings can result in distance compression (eg He et al 2004), so distance underestimation may have been expected underwater where optical cues are reduced. In the current experiment, participants viewed the same environment, so all optical cues were held constant across conditions. We examined whether changes in swimming ability would influence perceived distance underwater even when all optical information was held constant. We manipulated perceivers' abilities to move in the water by giving them swimming flippers or not, and found that swimmers judged the targets as closer when they wore the flippers and thus had enhanced swimming ability.

\section{Method}

\subsection{Participants}

Fifty-seven swimmers (twenty-one female) volunteered for the study. Participants were recruited at the pool during open swim hours and during the Purdue women's team swim practice (in which case, all the participants were on the swim team). The criteria for inclusion were at least some ability to swim and to have normal vision or wear contact lenses at the time of the study. These criteria resulted in the exclusion of one participant on the basis of very poor swimming ability and two participants on the basis of needing corrective lenses.

\subsection{Stimuli}

The study took place in the diving pool at the Boilermaker Aquatic Center. Three colored plastic discs (21 cm in diameter) served as targets. They were suspended approximately $0.5 \mathrm{~m}$ below the water surface with a clear fishing line and 3 weights placed on the bottom of the pool. Because the targets were not easy to reposition, they were set-up in the same location for every participant. The closest target was placed at $1.96 \mathrm{~m}$ at $45^{\circ}$ to the right of the participant, and was orange with a blue ring and black with an orange stripe on the back. The middle-distance target was placed at $3.18 \mathrm{~m}$ at $45^{\circ}$ to the left of the participant, and was blue with a yellow ring and black with a blue stripe on the back. The farthest target was placed at $4.39 \mathrm{~m}$ and set straight in front of the participant, and was green with an orange ring and black with a green stripe on the back. A pair of TYR Racetech goggles was used for estimating distance underwater.

\subsection{Procedure}

Participants were approached by the experimenter, who was also a certified life-guard and swim instructor with 6 years of competitive swimming experience. Participants first practiced estimating distances on the ground in order to get used to estimating distances. They stood at a designated spot on the side of the pool and estimated the distance to a wall that was $1.73 \mathrm{~m}$ away. They were told to imagine their body lying down and to imagine how many body lengths would fit in between them and the wall as a way to help them make estimates. Then they walked to another spot and estimated the distance to another wall that was $3.33 \mathrm{~m}$ away. Participants gave their estimates in their preferred unit of measurement. 
Participants were assigned to the flipper or no-flipper condition in alternating order. The condition specified whether or not they were wearing flippers when they estimated distances underwater, although all participants wore flippers at some point in the study. Participants were first exposed to the ease with which they could swim in each condition by swimming 4 lengths of the pool ( $9.6 \mathrm{~m}$ in length) in one condition and then another 4 lengths in the other condition. Those in the flipper condition first swam without the flippers then swam with the flippers so that the benefit of the flippers would be even more apparent. Those in the no-flipper condition first swam with the flippers and then swam without them so that the reduced ability without flippers would be more apparent. The participants were simply instructed what to do; they were not given a reason why they were wearing the flippers. While participants were swimming, the experimenter rated each participant on his/her ability to swim on a scale from 1 to 5. A ' 1 ' indicated that the participant had no ability to swim, and he/she was not included in the study. A ' 2 ' indicated that the participant had some ability to swim; a ' 3 ' indicated that he/she was a recreational swimmer; a '4' indicated that he/she was a non-active competitive swimmer; and a ' 5 ' indicated that the participant was an active competitive swimmer.

After exposure to the different swimming conditions, participants were instructed to swim to a set of submerged steps and to stand on the bottom step. From these steps, participants estimated the distance to the targets (see figure 1). Participants were asked to wear the provided set of goggles and to submerge their head completely underwater when viewing the targets. The order of the targets was randomized. For each target, participants first estimated how long it would take to swim to the target in seconds. This was done to get the participants to perceive the target in terms of swimming to it. Then, participants submerged their heads again and estimated the distance to the target in their preferred units of distance measurement. Participants were not restricted in the number of times they were allowed to go underwater to view the target, though one viewing was typically sufficient. This design allowed for a potential confound in that participants could have viewed the targets while their heads were out of the water. However, because we were interested in between-group differences, this confound was present for both conditions. Also, given the ripples on top of the water, vision of the targets was likely clearer underwater than when viewing just above the surface, so it is possible but unlikely that participants relied on information acquired when their heads were out of the water.

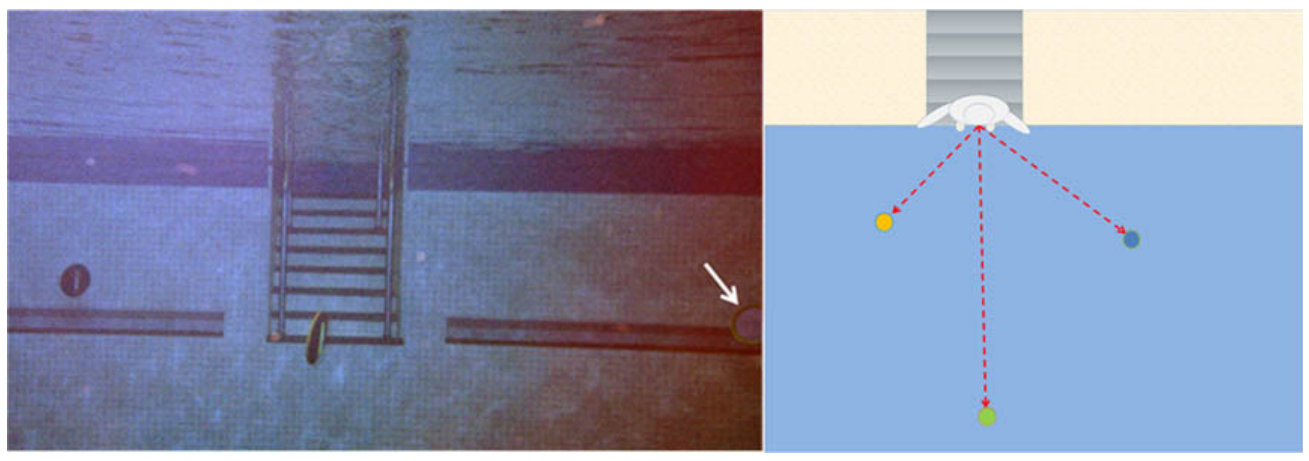

Figure 1. [In colour online, see http://dx.doi.org/10.1068/p6910] Underwater view (left) and bird's-eye view (right) of the experimental setup. Participants sat on the steps and estimated the distance to three submerged targets. The arrow points to one of the targets. 


\section{Results}

Participants were grouped according to their rated swimming ability. Those rated as a 4 or 5 were labeled as better swimmers and those rated as 2 or 3 were labeled as worse swimmers. $^{(1)}$

Participants who had better swimming ability judged the targets to be closer than did participants who had worse swimming ability (see figure 2). In addition, participants who were wearing swimming flippers and thus had enhanced ability to swim to targets judged the targets to be closer than did participants who had taken off the flippers and thus had reduced ability to swim to targets (see figure 3).

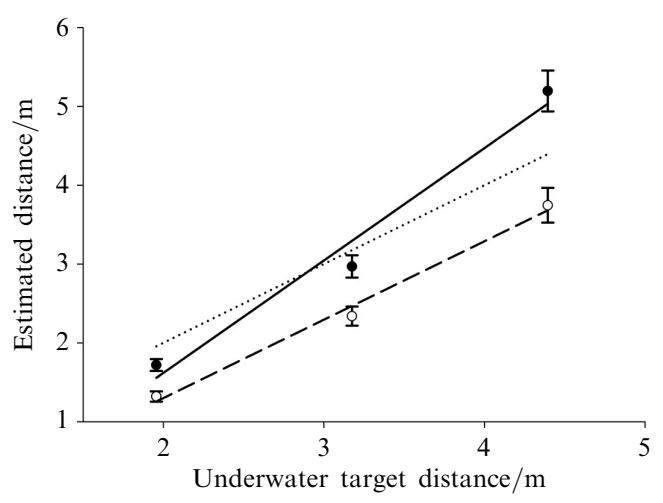

$\longrightarrow$ worse swimmers

Figure 2. Estimated distance to underwater targets as a function of target distance and whether the swimmer had better or worse swimming ability. Error bars represent 1 SEM. The dotted line represents perfect accuracy. The other lines represent linear regression for each group.

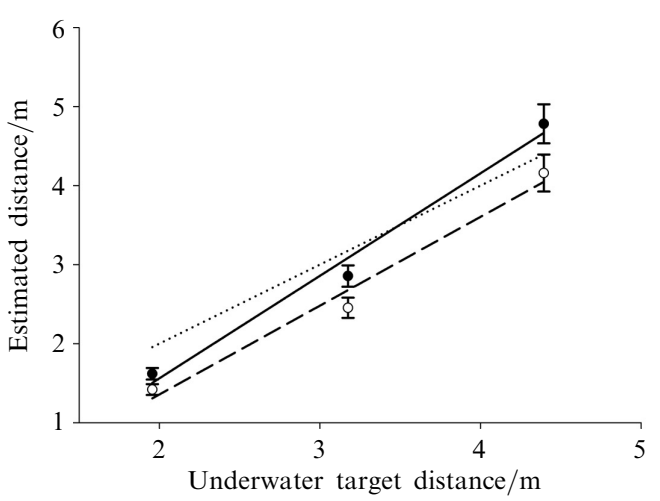

$\longrightarrow$ no flippers
$\ldots-$ - flippers

Figure 3. Estimated distance to underwater targets as a function of target distance and whether the swimmer was wearing flippers. Error bars represent $1 \mathrm{SEM}$. The dotted line represents perfect accuracy. The other lines represent linear regression for each condition.

These impressions were confirmed with a repeated-measures ANOVA with target distance as a repeated factor, flipper condition and ability group as between-subjects factors, and distance estimates as the dependent factor. Flipper condition significantly affected estimated distance $\left(F_{1,50}=4.89, p<0.05, \eta_{\mathrm{p}}^{2}=0.09\right)$. Participants wearing flippers judged the targets to be closer than did participants not wearing flippers. Ability of the group significantly affected estimated distance $\left(F_{1,50}=20.01, p<0.001\right.$, $\left.\eta_{\mathrm{p}}^{2}=0.29\right)$. Those with better swimming ability judged the targets to be closer than did those with worse swimming ability (see figure 3). There was not a significant interaction between flipper condition and swimming ability $\left(F_{1,50}=0.07, p>0.79\right.$; see figure 4). Target distance influenced estimated distance $\left(F_{2,100}=304.94, p<0.001\right.$,

(1) The ability groupings were based on an experienced but single experimenter's ratings. However, participants' estimated time to swim to the targets corroborated these ratings: the better swimmers estimated that it would take less time to swim to the targets $(M=2.01 \mathrm{~s}, \mathrm{SE}=0.32 \mathrm{~s})$ compared to the worse swimmers $(M=4.26 \mathrm{~s}, \mathrm{SE}=0.32 \mathrm{~s})\left(F_{1,50}=21.29, p<0.001, \eta_{\mathrm{p}}^{2}=0.30\right)$. Furthermore, the pattern of results was similar when participants were grouped according to whether they were currently on the swim team or not, and when they were grouped on the basis of a median split of their estimated time ratings to swim to the targets. 


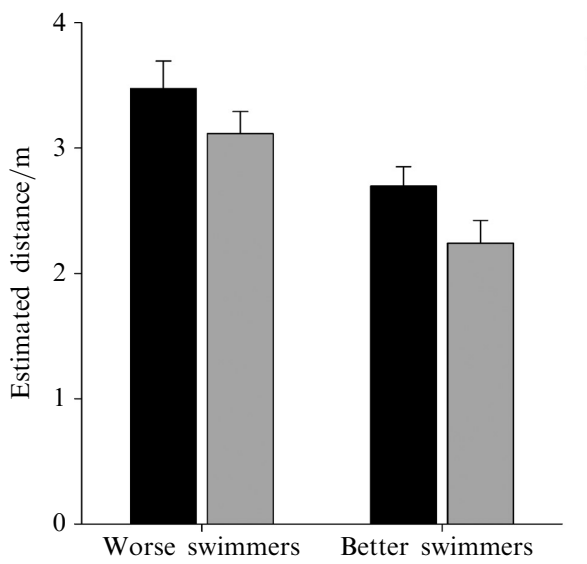

$\square$ no flippers
$\square$ flippers

Figure 4. Estimated distance to underwater targets as a function of swimming ability and flipper condition. Error bars represent 1 SEM.

$\left.\eta_{\mathrm{p}}^{2}=0.86\right)$. The interaction between target distance and flipper condition was not significant $\left(F_{2,100}=1.54, p>0.21\right)$. The interaction between target distance and ability of the group was significant $\left(F_{2,100}=10.50, p<0.001, \eta_{\mathrm{p}}^{2}=0.17\right)$. Those with worse swimming ability estimated the targets to be even farther as target distance increased. The three-way interaction between target distance, flipper condition, and swimming ability was not significant $\left(F_{2,100}=0.22, p>0.80\right)$.

Sex differences are also of potential interest given that women tend to be more buoyant. There were not enough women in our group of less-skilled swimmers $(n=2)$, so we could only look at sex differences in the more-skilled swimmers. While women $(M=2.22 \mathrm{~m}, \mathrm{SE}=0.13 \mathrm{~m}, n=18)$ did perceive the targets to be closer than men $\operatorname{did}(M=2.90 \mathrm{~m}, \mathrm{SE}=0.15 \mathrm{~m}, n=13)\left(F_{1,29}=11.24, p<0.01\right)$, the women were also rated as better swimmers $(M=4.94, \mathrm{SE}=0.09)$ than the men $(M=4.62 \mathrm{~m}$, $\mathrm{SE}=0.10 \mathrm{~m})\left(F_{1,29}=5.89, p<0.05\right)$. Thus, we are unable to decouple ability based on skill from effort based on buoyancy within the current sample.

\section{Discussion}

These results suggest that distance underwater is perceived in terms of the perceiver's ability to swim. Perceivers who anticipated being able to swim faster with less effort as a result of wearing the flippers judged the targets to be closer than did participants who took off the flippers. In addition, swimmers who were more skilled at swimming also judged the underwater targets to be closer than did those who were less skilled at swimming. Both findings suggest that ease to swim to a target influences the perceived distance to the target. The results also demonstrate that action-specific effects generalize to aquatic environments.

The action-specific perception account (see Proffitt and Linkenauger, in press; Witt 2011a), which proposes that perceivers see the surrounding environment in terms of their ability to act in it, is motivated by Gibson's (1979) ecological approach to perception. Gibson claimed that the main objects of perception are affordances, which are the possibilities for action. Here, we demonstrated that the affordance for swimming influences perceived distance underwater. That the same visual information looks different depending on one's ability challenges many notions of perception as providing a general-purpose and behaviourally neutral representation of the environment (eg Fodor 1983, pages 66-68; Loomis and Philbeck 2008; Pylyshyn 2006, pages $130-132)$. However, in addition to being consistent with ecological approaches (see Witt and Riley, submitted), the current results are also consistent with accounts of embodied cognition (eg Wilson 2002), common-coding approaches (Hommel et al 2001), 
and selection-for-action accounts (eg Bekkering and Neggers 2002). All of these approaches emphasize action in perceptual processes.

The current results expand on the previous literature by demonstrating actionspecific effects in an environment that differs from the one to which people have likely adapted. However, our perceptual systems evolved from systems that themselves evolved in aquatic and terrestrial environments, so one possibility is that these earlier perceptual systems were also tuned to the organisms' abilities. For example, frogs only see information from the environment that is relevant for their actions such as bugs, predators, and hiding places (Lettvin et al 1959). In their waggle dance, bees express distance in terms of the effort required to fly the specified distance, rather than in terms of the physical distance (von Frisch 1955, page 121). Ants that move more efficiently forage farther from their home than ants that move less efficiently (Nonacs 2002). Given that the current research demonstrates action-specific perception in an evolutionarily ancient environment, action-specific perceptual scaling may be a process shared by many organisms.

Though not mutually exclusive, another possibility is that, even though our perceptual systems did not specifically evolve to perceive underwater, we must rely on our terrestrially adapted perceptual system even when underwater. Given that this perceptual system involves action-specific processes, underwater distances will also be seen in terms of the perceiver's ability to achieve them so long as the perceptual system has access to information about the perceiver's abilities. If the perceptual system does not have access to information about the perceiver's ability to act, then action-specific scaling will not be apparent (Witt and Proffitt 2008). Thus, in a novel environment in which a perceiver has no action experience, action-specific scaling might not be apparent or might not be accurate relative to the perceiver's abilities. For safety reasons, we imposed the criterion that our current sample of participants must have a sufficient ability to swim. However, we would expect a different pattern of results for people who had never been exposed to swimming, because perceptual processes would not have access to information about the person's ability. For instance, an astronaut in a zero-gravity environment for the first time might not perceive the environment accurately relative to his/her zero-gravity abilities. With experience, however, the astronaut would become more tuned to his/her abilities and would then see the surrounding environment in terms of these abilities. As another example, in virtual environments perceived distance tends to be grossly underestimated relative to perceived distance in real environments (eg Loomis and Knapp 2003). Interestingly, perceived distance in virtual environments benefits from having a visually specified body compared to when the body is not specified (Mohler et al 2010). Thus, perceptual information about the perceiver's own body aids in perceiving distance (see Linkenauger et al 2010 for example with perceived size).

The current results suggest that action-specific perceptual effects can occur underwater. Swimmers with enhanced abilities due to wearing flippers perceived the targets to be closer than swimmers who were not wearing the flippers. More skilled swimmers also perceived the targets to be closer than less skilled swimmers. People perceive the surrounding environment in terms of their ability to act, and this action-specific scaling generalizes to multiple types of environments.

Acknowledgments. We would like to thank the Purdue swim club, the Purdue women's swim team, and head coach John Klinge; and the staff at the Boilermaker Aquatics Center, especially directors David Fraseur and Chris Crume. This work was supported by a grant from the National Science Foundation (BCS-0957051) to JKW. 


\section{References}

Bekkering H, Neggers S F W, 2002 "Visual search is modulated by action intentions" Psychological Science $13370-374$

Bhalla M, Proffitt D R, 1999 "Visual-motor recalibration in geographical slant perception" Journal of Experimental Psychology: Human Perception and Performance 25 1076-1096

Cañal-Bruland R, Kamp J van der, 2009 "Action goals influence action-specific perception" Psychonomic Bulletin \& Review $161100-1105$

Fodor J A, 1983 The Modularity of Mind (Cambridge, MA: MIT Press)

Frisch K von, 1955 The Dancing Bees: An Account of the Life and Senses of the Honey Bee (New York: Harcourt, Brace, and Co)

Gibson J J, 1979 The Ecological Approach to Visual Perception (Boston, MA: Houghton Mifflin)

Gutteling T P, Kenemans J L, Neggers S F W, 2011 "Grasping preparation enhances orientation change detection" PLoS ONE 6 e17675

He Z J, Wu B, Ooi T L, Yarbrough G, Wu J, 2004 "Judging egocentric distance on the ground: Occlusion and surface integration" Perception 33 789-806

Hommel B, Müsseler J, Aschersleben G, Prinz W, 2001 "The theory of event coding (TEC): A framework for perception and action planning" Behavioural and Brain Sciences 24 849-937

Lessard D A, Linkenauger S A, Proffitt D R, 2009 "Look before you leap: Jumping ability affects distance perception" Perception 381863 - 1866

Lettvin J Y, Maturana H R, McCulloch W S, Pitts W H, 1959 "What the frog's eye tells the frog's brain" Proceedings of the IRE 471941 - 1951

Linkenauger S A, Ramenzoni V, Proffitt D R, 2010 "Illusory shrinkage and growth: body-based rescaling affects the perception of size" Psychological Science $211318-1325$

Linkenauger S A, Witt J K, Proffitt D R, in press "Taking a hands-on approach: Apparent grasping ability scales the perception of object size" Journal of Experimental Psychology: Human Perception and Performance

Linkenauger S A, Witt J K, Stefanucci J K, Bakdash J Z, Proffitt D R, 2009 "The effect of handedness and reachability on perceived distance" Journal of Experimental Psychology: Human Perception and Performance 351649 - 1660

Loomis J M, Knapp J M, 2003 "Visual perception of egocentric distance in real and virtual environments", in Virtual and Adaptive Environments Eds L J Hettinger, M W Haas (Mahwah, NJ: Lawrence Erlbaum Associates) pp 21-46

Loomis J M, Philbeck J W, 2008 "Measuring spatial perception with spatial understanding and action", in Embodiment, Ego-Space, and Action Eds R L Klatzky, B MacWhinney, M Behrmann (Cambridge, MA: MIT Press) pp 1-44

Luria S M, Kinney J A, 1970 "Underwater vision" Science 167 1454-1461

Luria S M, Kinney J A, Weissman S, 1967 "Estimates of size and distance underwater" American Journal of Psychology $\mathbf{8 0} 282-286$

Mohler B J, Creem-Regehr S H, Thompson W B, Bülthoff H B, 2010 "The effect of viewing a selfavatar on distance judgments in an HMD-based virtual environment" Presence: Teleoperators and Virtual Environments $19230-242$

Nonacs P, 2002 "Patterns of energy allocation within foragers of Formica planipilis and Pogonomyrmex salinus" Western North American Naturalist 62188 - 196

Proffitt D R, Linkenauger S A, in press, "Perception viewed as a phenotypic expression", in Tutorials in Action Science Eds W Prinz, M Beisert, A Herwig (Cambridge, MA: MIT Press)

Proffitt D R, Stefanucci J K, Banton T, Epstein W, 2003 "The role of effort in distance perception" Psychological Science $14106-112$

Pylyshyn Z W, 2006 Seeing and Visualizing: It's Not What You Think (Cambridge, MA: MIT Press)

Ross H E, 1967 "Water, fog and the size-distance invariance hypothesis" British Journal of Psychology $58301-313$

Stefanucci J K, Proffitt D R, Banton T, Epstein W, 2005 "Distances appear different on hills" Perception \& Psychophysics $671052-1060$

Symes E, Tucker M, Ellis R, Vainio L, Ottoboni G, 2008 "Grasp preparation improves change detection for congruent objects" Journal of Experimental Psychology: Human Perception and Performance $34854-871$

Wilson M, 2002 "Six views of embodied cognition" Psychonomic Bulletin \& Review 9 625-636

Witt J K, 2011a "Action's effect on perception" Current Directions in Psychological Science 20 $201-206$

Witt J K, 2011b "Tool use influences perceived shape and parallelism: Indirect measures of perceived distance" Journal of Experimental Psychology: Human Perception and Performance 37 available online at doi:10.1037/a0021933 
Witt J K, Dorsch T, 2009 "Kicking to bigger uprights: Field goal kicking performance influences perceived size" Perception 381328 - 1340

Witt J K, Proffitt D R, 2005 "See the ball, hit the ball: Apparent ball size is correlated with batting average" Psychological Science $16937-938$

Witt J K, Proffitt D R, 2008 "Action-specific influences on distance perception: A role for motor simulation" Journal of Experimental Psychology: Human Perception and Performance 34 1479-1492

Witt J K, Proffitt D R, Epstein W, 2004 "Perceiving distance: A role of effort and intent" Perception $33570-590$

Witt J K, Proffitt D R, Epstein W, 2005 "Tool use affects perceived distance but only when you intend to use it" Journal of Experimental Psychology: Human Perception and Performance 31 $880-888$

Witt J K, Proffitt D R, Epstein W, 2010 "How and when does action scale perception?" Journal of Experimental Psychology: Human Perception and Performance $361153-1160$

Witt J K, Riley M, submitted, "Getting in touch with your inner Gibson" The Journal of Mind and Behavior

Witt J K, Sugovic M, 2010 "Performance and ease influence perceived speed" Perception 391341 - 1353 